\title{
Ionization Correction Factors from an Extensive Grid of Photoionization Models of Planetary Nebulae
}

\author{
O. Rokach, V. Golovaty
}

Astronomical Observarory of Lviv State University, Ukraine

Using a new photoionization code PAN (Yu.Malkov et al, this book) we have built a grid of 250 photoionization models for planetary nebulae (PN). The stellar evolutionary tracks for PN nuclei with $M_{*}=0.566,0.598,0.644$ and $0.8 M_{\odot}$ are taken from the computations of the Blöcker $(A \& A, 299,1995)$. On the $L_{*}-T_{\text {eff }}$ diagram our grid covers whole region in which the PN nuclei are observed. The spectra of the central stars at $\lambda \leq 912 \AA$ corresponded to non-LTE model atmospheres (R.E.S. Clegg and D. Middlemass, MNRAS, 228, 1987), corrected for presence of the stellar wind. The nebular shell was assumed to be spherically symmetric, and the profile of the radial gas density distribution in it was taken equal to the empirical approximate expression found from the analysis of isophotal maps of real PN (V. Golovaty and Yu. Malkov, Astron. Zh., 69, 1992). Each model was calculated for three choises of $M_{s h e l l}$ and each of them for two choises of shell expansion velosity 15 and $30 \mathrm{~km} / \mathrm{sec}$ respectively. The relative elemental abundances were the same for all models: H:He:C:N:O:Ne:Mg:Si:S:Ar = 10000:1200:5.0:1.0:6.0:0.8:0.3:0.3:0.15:0.04 (by number of atoms).

Using a method of "intersection of curves" for four ionization zones (OI, OII, OIII and HeII) we determined the electron density $n_{e}$ and electron temperature $T_{e}$ for each of the calculated models. The values found for $n_{e}$ and $T_{e}$ in this way were used for the determination of the ionic relative abundances in each zone.

Using least squares method we investigate dependences of type $\log \left[\left(A^{+i} / H^{+}\right) /(A /-\right.$ $H)]=f(\mathrm{x})$ and $\log \left[\left(A^{+i} / H^{+}\right) /(A / H)\right]=f(\mathrm{x}, \mathrm{y}), \mathrm{x}=\log \left[X^{+k+1} / X^{k}\right], \mathrm{y}=\log \left[Y^{+k+1} / Y^{k}\right]$, where $A / H$ is the abundance of some element $\mathrm{A}, A^{+i} / H^{+}$is the relative abundance of the $i$-th stage of ionization of that element, $X^{+k+1} / X^{k}$ and $Y^{+k+1} / Y^{k}$ is one of the ratios $\mathrm{He}^{++} / \mathrm{He}^{+}, \mathrm{O}^{++} / \mathrm{O}^{+}, \mathrm{O}^{+3} / \mathrm{O}^{++}, \mathrm{Ne} e^{++} / \mathrm{Ne}^{+}, \mathrm{S}^{++} / \mathrm{S}^{+}, \mathrm{S}^{+3} / \mathrm{S}^{++}$or $\mathrm{Ar}^{+3} / \mathrm{Ar}^{++}$. Such dependences enable us to obtain the total abundance $A / H$ of the element from the abundance $A^{+i} / H^{+}$of an ion, i.e. to allow for unobservable stages of ionization of element A. 